\title{
Topological Impurity Segregation at Faceted Silicon Grain Boundaries Studied by Correlative Atomic-Resolution STEM and APT
}

\author{
CH Liebscher ${ }^{1}$, A Stoffers ${ }^{1,2}$, O Cojocaru-Mirédin ${ }^{2}$, B Gault ${ }^{1}$, C Scheu $^{1}$, G Dehm $^{1}$ and D Raabe ${ }^{1}$ \\ 1. Max-Planck-Institut für Eisenforschung GmbH, 40237 Düsseldorf, Germany. \\ 2. RWTH Aachen, Institute of Physics (IA), 52056 Aachen, Germany.
}

Since the end of the $19^{\text {th }}$ century the detrimental effect of trace elements on the macroscopic materials properties has been observed [1]. However, the connection to the underlying microstructure was not possible to be resolved at that time. In the past two decades, our understanding of the interplay of lattice defects and local compositional modulations has dramatically changed. Especially the development of atomic resolution microscopy techniques established an unprecedented view into the chemistry and atomic structure of defects and interfaces.

Most modern energy related materials are polycrystalline and hence internal interfaces such as grain boundaries largely dominate the macroscopic material behavior. Grain boundaries are separating two crystals with different orientation and are typically regarded as planar defects. However, recent atomic scale investigations confirmed structural transitions at these interfaces and thus the grain boundary itself can behave like a two-dimensional (2D) phase [2]. Furthermore, roughening or faceting transitions of the boundaries add a topological aspect to the description and properties of grain boundaries [3].

In multicrystalline Silicon (mc-Si), coincident site lattice (CSL) boundaries and trace impurities are degrading the electrical performance by promoting charge carrier recombination [4]. Our study gives an unprecedented view in the connection of the grain boundary structure and the related impurity segregation. The direct correlation of atomic resolution aberration-corrected scanning transmission electron microscopy (STEM) and 3D atom probe tomography unravels a topological segregation of Carbon $(\mathrm{C})$ and Iron $(\mathrm{FeN})$ at linear junctions of merging grain boundary facets. The facets are composed of alternating coherent and incoherent grain boundary segments observed for both $\Sigma 3$ (Fig. 1a) and $\Sigma 9$ (Fig. 2a) grain boundaries. The corresponding solute segregation of $\mathrm{C}$ and $\mathrm{FeN}$ for the same faceted $\Sigma 3$ grain boundary is illustrated in Fig. 1b. It is obvious that both $\mathrm{C}$ and $\mathrm{FeN}$ are confined to regularly spaced, tubular regions that almost perfectly resemble the topological grain boundary structure.

The proof of this topological segregation phenomenon is established by observing the atomic structure of a $\Sigma 9$ grain boundary on an APT needle by STEM and a subsequent 3D chemical analysis of the same specimen in the atom probe. The corresponding STEM images and 3D APT reconstruction of C and $\mathrm{FeN}$ are illustrated in Fig. 2. The topology of the grain boundary follows the same sequence of alternating coherent and incoherent segments with length between $10 \mathrm{~nm}$ and $50 \mathrm{~nm}$. The superimposed APT reconstruction on an annular bright-field (ABF) STEM image of the APT tip confirms the segregation of $\mathrm{C}, \mathrm{Fe}$ and $\mathrm{N}$ to the line segment of two merging grain boundary facets. An outlook on the involved mechanisms leading to this novel segregation phenomenon will be given and the influence of the local strain state at the facet junctions will be discussed in detail. 


\section{References:}

[1] WC Robertsausten, Proceedings of the Royal Society of London 43 (1887), p. 425.

[2] T Frolov et al, Nature Communications 4 (2013), p. 1899.

[3] AP Sutton and RW Balluffi, Acta Metallurgica 35 (1987), p. 2177.

[4] A Stoffers et al, Prog. Photovolt: Res. Appl. 23 (2015), p. 1742.
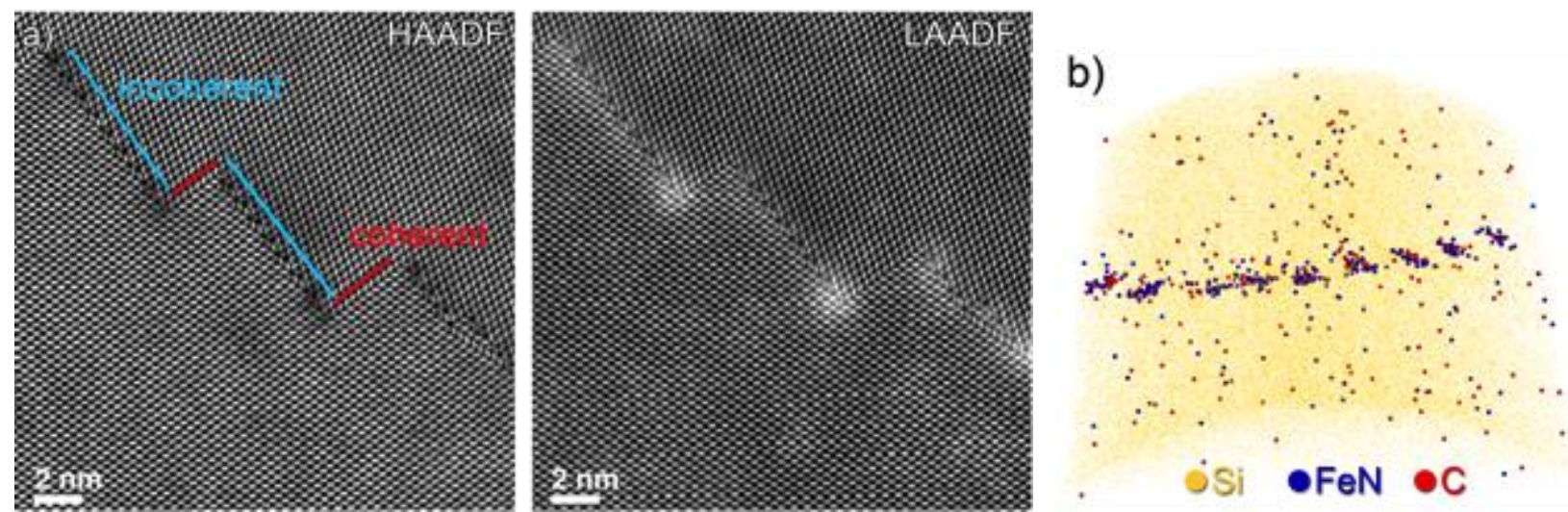

Figure 1. a) Aberration-corrected HAADF- and LAADF-STEM images of a faceted $\Sigma 3$ grain boundary in mc-Si. b) 3D APT reconstruction of Silicon (Si), Carbon (C) and Iron (FeN) from the same grain boundary.
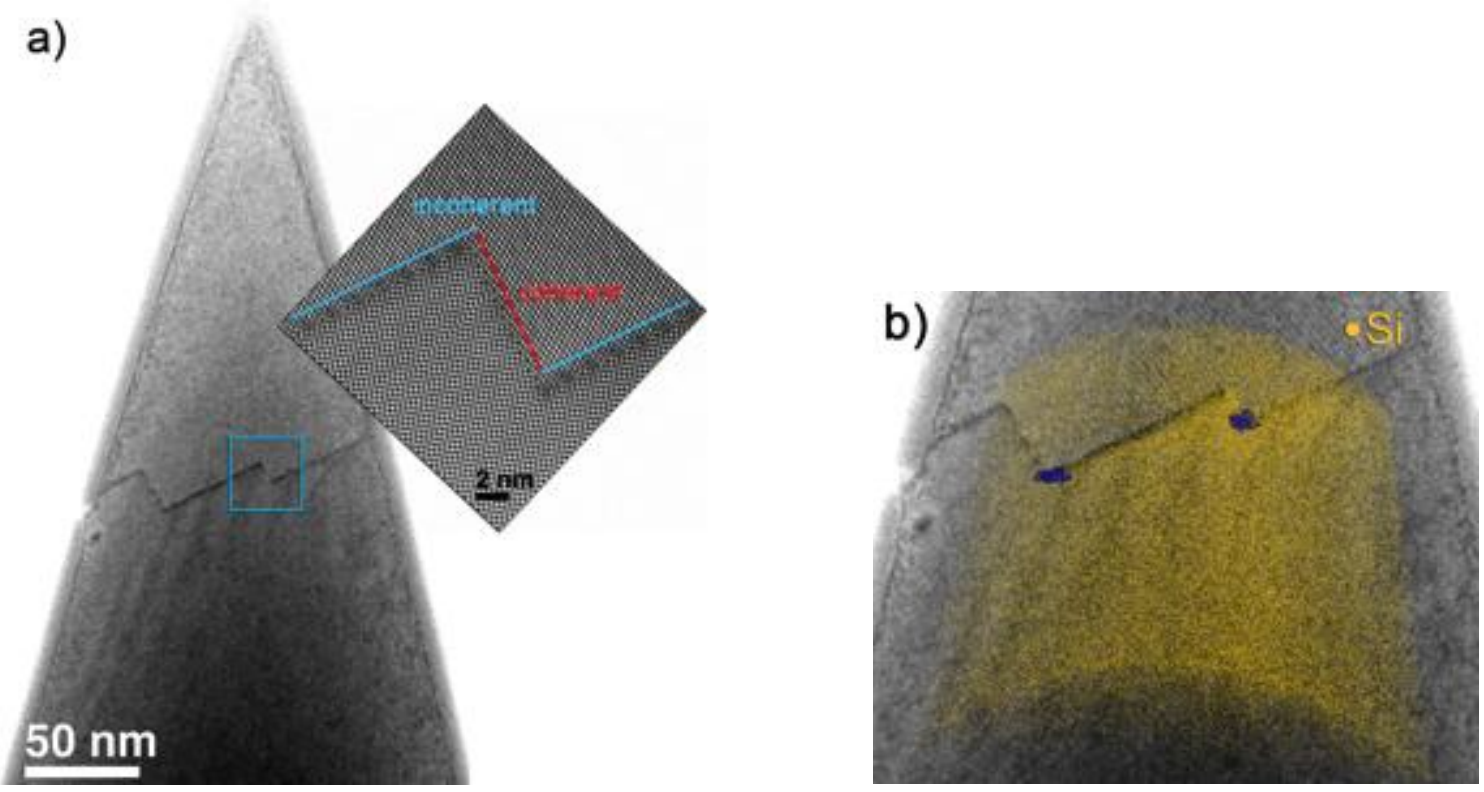

Figure 2. a) ABF-STEM overview image of an APT needle with a magnified region of the grain boundary facets imaged under HAADF conditions. b) 3D atom probe tomogram superimposed on the ABF-STEM image of the APT needle. The blue regions represent the isodensity surfaces of $\mathrm{C}+\mathrm{FeN}$ for $0.15 \mathrm{at} / \mathrm{nm}^{3}$. 